\title{
A REMAINDER FORMULA AND LIMITS OF CARDINAL SPLINE INTERPOLANTS
}

BY

T. N. T. GOODMAN AND S. L. LEE

ABSTRaCt. A Peano-type remainder formula

$$
f(x)-S_{n}(f ; x)=\int_{-\infty}^{\infty} K_{n}(x, t) f^{(n+1)}(t) d t
$$

for a class of symmetric cardinal interpolation problems C.I.P. $(E, F, \mathbf{x})$ is obtained, from which we deduce the estimate $\left\|f-S_{n, r}(f ;)\right\|_{\infty} \leqslant K\left\|f^{(n+1)}\right\|_{\infty}$. It is found that the best constant $K$ is obtained when $\mathbf{x}$ comprises the zeros of the EulerChebyshev spline function. The remainder formula is also used to study the convergence of spline interpolants for a class of entire functions of exponential type and a class of almost periodic functions.

1. Introduction. As in [5], for $\mathbf{x}=\left(x_{0}, x_{1}, \ldots, x_{m}\right), 0=x_{0}<x_{1}<\cdots<x_{m}=1$ and incidence matrices $E=\left\|E_{i j}\right\|_{i=0}^{m}{ }_{i=0}^{n}, F=\left\|F_{i j}\right\|_{i=0 j=0}^{m}$ with $E_{0 j}=E_{m j}$ and $F_{0 j}=$ $F_{m j}, j=0, \ldots, n$, let $\bigodot(F, \mathbf{x}):=\left\{f: \mathbf{R} \rightarrow \mathbf{C} ; \forall \nu \in \mathbf{Z}, f \mid\left(\nu+x_{i}, \nu+x_{i+1}\right) \in \pi_{n}\right.$, $i=0, \ldots, m-1$, and $f^{(n-j)}\left(\nu+x_{i}^{-}\right)=f^{(n-j)}\left(\nu+x_{i}^{+}\right) \forall(i, j)$ with $\left.F_{i j}=0\right\}$, and refer to the following 'cardinal' interpolation problem as the C.I.P. $(E, F, \mathbf{x})$ :

For sequences of numbers $\left\{y^{(i, j)}\right\}=\left\{y_{\nu}^{(i, j)} ; 0 \leqslant i<m\right.$ and $\left.E_{i j}=1\right\}$, find $S \in$ $\mathrm{e}(F, x)$ satisfying $S^{(j)}\left(\nu+x_{i}\right)=y_{\nu}^{(i, j)}$.

Sufficient conditions for C.I.P. $(E, F, \mathbf{x})$ to be poised, i.e. existence of a unique $S \in \mathcal{C}(F, \mathbf{x}), S(x)=O\left(|x|^{\gamma}\right)$ as $x \rightarrow \pm \infty$ satisfying $S^{(j)}\left(\nu+x_{i}\right)=y_{\nu}^{(i, j)}$ when $y_{\nu}^{(i, j)}$ $=O\left(|\nu|^{\gamma}\right)$, are given in [5].

Suppose that the C.I.P. $(E, F, \mathbf{x})$ is poised; then given a sufficiently smooth function $f$ of power growth $\exists$ a unique $S_{n}(f ;) \in \mathcal{C}(F, \mathbf{x})$ of power growth which interpolates $f$ in the sense that

$$
S_{n}^{(j)}\left(f ; \nu+x_{i}\right)=f^{(j)}\left(\nu+x_{i}\right), \quad \nu \in \mathbf{Z}, E_{i j}=1 .
$$

The following problem then arises.

Problem. Find necessary and sufficient conditions so that $S_{n}(f ;) \rightarrow f$ uniformly as $n \rightarrow \infty$.

This question was first raised by Schoenberg [9] who also found a sufficient condition for the convergence of $S_{n}(f ;)$ for the case where $m=1, n$ is odd, $E=F$ and

$$
E_{0 j}=E_{1 j}= \begin{cases}0, & j=1,2, \ldots, n \\ 1, & j=0\end{cases}
$$

Received by the editors February 20, 1980 and, in revised form, March 23, 1981.

1980 Mathematics Subject Classification. Primary 41A15, 41A05. 
In this case $\mathcal{E}(F, \mathbf{x})$ comprises odd degree cardinal splines with integer knots. Schoenberg [9] proves the following

THEOREM A. Let

$$
f(x)=\frac{1}{2 \pi} \int_{-\pi}^{\pi} e^{i u x} d \alpha(u)
$$

where $\alpha(u)$ is a function of bounded variation in $[-\pi, \pi]$ and let $A=\alpha(-\pi+0)-$ $\alpha(-\pi), B=\alpha(\pi)-\alpha(\pi-0)$. Then

$$
\lim _{k \rightarrow \infty} S_{2 k-1}(f ; x)=f(x)+\frac{i(A-B)}{2 \pi} \sin \pi x
$$

uniformly on $\mathbf{R}$.

A partial converse of Theorem A was given by Richards and Schoenberg [8]. Subsequently, Marsden and Riemenschneider [6] generalised Theorem A to the case of cardinal Hermite interpolation which corresponds to $m=1, E=F$ and, for some $1 \leqslant r \leqslant \frac{1}{2}(n+1)$

$$
E_{0 j}=E_{1 j}= \begin{cases}1, & j=0,1, \ldots, r-1, \\ 0, & j=r, \ldots, n .\end{cases}
$$

In this case, a sufficient condition for convergence of $S_{n}(f ;)$ analogous to that of Richards and Schoenberg was given by Goodman [4].

Recently, in an attempt to obtain more information on the convergence problem, I. J. Schoenberg [10] obtained a Peano type remainder formula

$$
f(x)-S_{2 k-1}(f ; x)=\int_{-\infty}^{\infty} K_{2 k-1}(x ; t) f^{(2 k)}(t) d t \quad(x \in \mathbf{R})
$$

for the case $m=1, n=2 k-1, E=F$ and $E$ satisfies (1.2), where $K_{2 k-1}(x, t):=(x-t)_{+}^{2 k-1}-S_{2 k-1}\left((\cdot-t)_{+}^{2 k-1} ; x\right)$. From (1.4), Schoenberg [10] deduced Theorem $\mathrm{A}$ and also obtained a convergence result for a class of almost periodic functions.

In this paper we shall consider the symmetric C.I.P. $(E, F, \mathbf{x})$

$$
\begin{aligned}
x_{i} & =1-x_{m-i}, \quad i=0, \ldots, m, \\
F_{i j} & =1 \quad \text { iff } i=0 \text { or } m \text { and } j=0, \ldots, r-1,
\end{aligned}
$$

for some $1 \leqslant r \leqslant n+1$, and either

(a) $n+r$ is even, $m=r$ and $E_{i j}=1$ iff $j=0$ and $i=0, \ldots, m$, or

(b) $n+r$ is odd, $m=r+1$ and $E_{i j}=1$ iff $j=0$ and $i=1, \ldots, m-1$.

That this problem is poised follows from Corollary 4.3 of [5] and was earlier shown by Micchelli [7]. In this case the class of cardinal spline functions $\varrho(F, \mathbf{x})$ is usually denoted by $\varsigma_{n, r}$, and clearly

$$
\varsigma_{n, r} \equiv \mathcal{C}(F, \mathbf{x})=\left\{S \in C^{n-r}(\mathbf{R}) ;\left.S\right|_{(\nu, \nu+1)} \in \pi_{n}, \forall \nu \in \mathbf{Z}\right\} .
$$

For $r, n$ as above, we define $\mathscr{F}_{n, r}:=\left\{f \in C^{n+1-r}(\mathbf{R}) ;\left.f\right|_{(\nu, \nu+1)} \in C^{n}[(\nu, \nu+1)]\right.$ and $f^{(n)}$ bounded and absolutely continuous on $\left.(\nu, \nu+1), \forall \nu \in \mathbf{Z}\right\}$. 
For $f \in \mathscr{F}_{n, r}$ of power growth, we let $S_{n, r}(f ;)$ denote the unique function of power growth that interpolates $f$ as in (1.1). Following the approach of Schoenberg [10] we derive a formula for the remainder $f-S_{n, r}(f ;)$ and deduce the following result.

THEOREM 1. For fixed $n, r$ and $\mathbf{x}, \exists K$ such that for any $f \in \mathscr{F}_{n, r}$ with $f^{(n+1-r)}$ of power growth and $\left\|f^{(n+1)}\right\|_{\infty}<\infty$,

$$
\left\|f-S_{n, r}(f ;)\right\|_{\infty} \leqslant K\left\|f^{(n+1)}\right\|_{\infty}
$$

and equality is attained for some $f \in \mathcal{F}_{n, r}$. For fixed $n, r, K$ is a minimum when $\mathbf{x}$ comprises the zeros of the Euler-Chebyshev spline $\mathcal{E}_{n+1, r}$ (see [1] and [4]) and in this case equality is attained for $f=\varepsilon_{n+1, r}$.

For $r=n+1$, this result reduces to a classical result on optimal constants in the remainder for Lagrange interpolation by polynomials (see [3, p. 64]).

Now let $B_{\sigma}=\{f ; f$ is the restriction to $\mathbf{R}$ of an entire function of exponential type $\leqslant \sigma$ and $\left.\|f\|_{\infty}<\infty\right\}$.

By deriving bounds on the best constants $K$ in (1.6), we prove the following results, all of which are generalisations of results of Schoenberg [10].

THEOREM 2. For fixed $\mathbf{x}$ and $r \geqslant 1, \exists K_{r}$ such that for all $n \geqslant r-1$ and $f \in B_{\sigma}$,

$$
\left\|f-S_{n, r}(f ;)\right\|_{\infty} \leqslant K_{r}(\sigma / r \pi)^{n+1}\|f\|_{\infty} .
$$

THEOREM 3. If $f(x)=\int_{-r \pi}^{r \pi} e^{i u x} d \alpha(u)$, where $\alpha(u)$ is a function of bounded variation in $[-r \pi, r \pi]$, then

$$
\lim _{n \rightarrow \infty} S_{n, r}(f ; x)=f(x)+C 2^{r-1} \prod_{i=1}^{r} \sin \pi\left(x-x_{i}\right)
$$

uniformly, where

$$
C= \begin{cases}(-1)^{r} i\{\alpha(r \pi)-\alpha(r \pi-0)+\alpha(-r \pi)-\alpha(-r \pi+0)\} & \text { if } n+r \text { is even } \\ (-1)^{r-1}\{\alpha(r \pi)-\alpha(r \pi-0)-\alpha(-r \pi)+\alpha(-r \pi+0)\} & \text { if } n+r \text { is odd }\end{cases}
$$

THEOREM 4. If $f \in B_{r \pi}$ is almost periodic in the sense of Bohr, then

$$
\lim _{n \rightarrow \infty} S_{n, r}(f ; x)=f(x)+C 2^{r} \prod_{i=1}^{r} \sin \pi\left(x-x_{i}\right)
$$

uniformly, where

$$
C= \begin{cases}(-1)^{r} \lim _{T \rightarrow \infty} \frac{1}{T} \int_{0}^{T} f(x) \sin r \pi x d x & \text { if } n+r \text { is even } \\ (-1)^{r-1} \lim _{T \rightarrow \infty} \frac{1}{T} \int_{0}^{T} f(x) \cos r \pi x d x & \text { if } n+r \text { is odd }\end{cases}
$$

In $\$ 2$ we apply the results of our preceding paper [5] to the study of the sign structure of the kernel, from which the corresponding remainder formula is derived. The proof of Theorem 1 is given in $\S 3$. Theorem 1 is then used in $\S 4$ to derive convergence results. 
2. The remainder formula. Take $1 \leqslant r \leqslant n+1$ and consider the poised, symmetric C.I.P. $(E, F, \mathbf{x})$ defined in $\S 1$. In this case the relation (1.5) and the conditions (a) and (b) imply that $0<x_{1}<\cdots<x_{r} \leqslant 1$ with equality iff $n+r$ is even. We shall also write $\mathcal{S}_{n, r}$ for $\mathcal{E}(F, \mathbf{x})$ and $\tilde{S}_{n, r}$ for $\mathcal{E}(E, \mathbf{x})$ which corresponds to the dual C.I.P. $(F, E, \mathbf{x})$.

Specialising the results of [5] to this special case it is easy to see that the corresponding null space

$$
\varrho^{0} \equiv \varrho^{0}(F, \mathbf{x}):=\left\{S \in \varrho(F, \mathbf{x}) ; S\left(\nu+x_{i}\right)=0 \forall \nu \in \mathbf{Z}, E_{i 0}=1\right\}
$$

has dimension $d$, where

$$
d= \begin{cases}n-\dot{r} & \text { if } n+r \text { is even, } \\ n-r+1 & \text { if } n+r \text { is odd }\end{cases}
$$

and is spanned by $d$ eigensplines $S_{j}, j=1, \ldots, d$, satisfying the functional relation

$$
S_{j}(x+1)=\lambda_{j} S_{j}(x) \quad \forall x \in \mathbf{R} .
$$

The eigenvalues $\lambda_{j}, j=1, \ldots, d$, of the C.I.P. $(E, F, \mathbf{x})$ are real, distinct, of $\operatorname{sign}(-1)^{r}$ and are precisely the eigenvalues of the matrix $C=\left(C_{\mu \nu}\right)_{d \times d}$ where $S^{\mu}(1)=$ $C_{\mu \nu} S^{(\nu)}(0), \mu, \nu=0, \ldots, d$.

Now for $i=1, \ldots, r$, we let $L_{i}$ denote the unique element of $\mathcal{C}(F, \mathbf{x})$ of power growth (actually of exponential decay) satisfying

$$
L_{i}\left(\nu+x_{j}\right)=\delta_{\nu 0} \delta_{i j}, \quad \forall \nu \in \mathbf{Z}, j=1, \ldots, r .
$$

Then, for $f \in \widetilde{F}_{n, r}$ of power growth, the unique function $S_{n, r}(f ;) \in \mathcal{C}(F, \mathbf{x})$ of power growth that interpolates $f$ for the C.I.P. $(E, F, \mathbf{x})$ is given by

$$
S_{n, r}(f ; x)=\sum_{i=1}^{r} \sum_{\nu=-\infty}^{\infty} f\left(\nu+x_{i}\right) L_{i}(x-\nu) .
$$

We let $\tilde{S}_{n, r}(f ;) \in \tilde{S}_{n, r}$ denote the unique spline function of power growth that interpolates $f$ for the dual C.I.P. $(F, E, \mathbf{x})$.

For $x, t \in \mathbf{R}$ we define

$$
g_{t}(x) \equiv \tilde{g}_{x}(t):=(1 / n !)(x-t)_{+}^{n}
$$

and

$$
K(x, t) \equiv K_{t}(x) \equiv \tilde{K}_{x}(t):=g_{t}(x)-S_{n, r}\left(g_{t} ; x\right) .
$$

Clearly, from (2.1), we have

$$
K(x+1, t+1)=K(x, t), \quad \forall x, t \in \mathbf{R},
$$

and

$$
K_{t}\left(\nu+x_{i}\right)=0, \quad \forall \nu \in \mathbf{R} \text { and } i=1, \ldots, r .
$$

Now we see from (2.2) and (2.3) that, for $\rho=0, \ldots, n$,

$$
\tilde{K}_{x}^{(\rho)}(t)=(-1)^{\rho} g_{t}^{(\rho)}(x)-S_{n, r}\left((-1)^{\rho} g_{t}^{(\rho)} ; x\right) .
$$

But for $\nu \in \mathbf{Z}$ and $\rho=0, \ldots, r-1, g_{\nu}^{(\rho)} \in \mathcal{C}(F, \mathbf{x})$ and so

$$
\tilde{K}_{x}^{(\rho)}(\nu)=0, \quad \forall x \in \mathbf{R} \text {. }
$$


Now we see from (2.2) that, for fixed $x, S_{n, r}\left(g_{i} ; x\right)$ as a function of $t$ lies in $\mathcal{C}(E, \mathbf{x})$ and has power growth. By (2.6) it interpolates $\tilde{g}_{x}$ for the C.I.P. $(F, E, \mathbf{x})$. Hence $S_{n, r}\left(g_{t} ; x\right)=\tilde{S}_{n, r}\left(\tilde{g}_{x} ; t\right), \forall x, t \in \mathbf{R}$, and so

$$
K(x, t)=\tilde{g}_{x}(t)-\tilde{S}_{n, r}\left(\tilde{g}_{x} ; t\right) .
$$

LEMMA 2.1. For $1<r<n$, there is a constant $\beta>0$ such that the following hold for $\rho=0, \ldots, n$.

(a) For $t \in \mathbf{R}, K_{t}^{(\rho)}(x)=O\left(e^{-\beta|x|}\right)$ as $x \rightarrow \pm \infty$ and the only zeros of $K_{t}(t \notin \mathbf{Z})$ are simple zeros at $\nu+x_{i}, \nu \in \mathbf{Z}, i=1, \ldots, r$.

(b) For $x \in \mathbf{R}, \tilde{K}_{x}^{(\rho)}(t)=O\left(e^{-\beta|t|}\right)$ as $t \rightarrow \pm \infty$ and the only zeros of $\tilde{K}_{x}(x \notin \mathbf{Z}+$. $\left.x_{i}\right)$ are isolated zeros of multiplicity $r$ at the integers.

Proof. We shall prove only (a) as (b) follows similarly by duality, and only for even $n+r$ as the result for odd $n+r$ follows similarly. By (2.4) and (2.6) we may suppose $0<t<1$.

Now, for $n+r$ even the C.I.P. $(E, F, \mathbf{x})$ has $n-r$ distinct eigenvalues $\lambda_{1}, \ldots, \lambda_{n-r}$ of sign $(-1)^{r}$ with $\left|\lambda_{1}\right|>\left|\lambda_{2}\right|>\cdots>\left|\lambda_{n-r}\right|>0$ and $\lambda_{n-r-i+1}=\lambda_{i}^{-1}, i=1, \ldots$, $n-r$.

We let $S_{1}, \ldots, S_{n-r}$ denote the corresponding eigensplines, which span $\mathcal{C}^{0}$. The eigenvalues $\lambda_{1}, \ldots, \lambda_{n-r}$ are precisely the eigenvalues of the matrix $C$ where $(-1)^{r} C$ is an oscillation matrix with corresponding eigenvectors $\left(S_{i}^{\prime}(0), \ldots, S_{i}^{(n-r)}(0)\right), i=$ $1, \ldots, n-r$. So by a theorem of Gantmacher and Krein we have

$$
S\left(S_{i}^{\prime}(0), \ldots, S_{i}^{(n-r)}(0)\right)=i-1, \quad i=1, \ldots, n-r
$$

(see also Micchelli [7]).

Now $K_{t} \in C^{n-r}(\mathbf{R})$ and for $\nu=1,2, \ldots,\left.K_{t}\right|_{(\nu, \nu+1)} \in \pi_{n}$. Thus, by $(2.5),\left.K_{t}\right|_{(1, \infty)}$ can be extended to an element of $e^{0}$. Since $K_{t}$ is of power growth, we therefore have

$$
K_{t}(x)=\sum_{i \geqslant p} c_{i} S_{i}(x), \quad \forall x \geqslant 1,
$$

where $c_{p} \neq 0$ and $p>\frac{1}{2}(n-r)$. Similarly $K_{t}(x)=\sum_{i \leqslant q} c_{i} S_{i}(x), \forall x \leqslant 0$, where $c_{q} \neq 0$ and $q<\frac{1}{2}(n-r)$.

The first part of (a) follows.

Now for $\rho=0, \ldots, n-r$ and $\nu=1,2, \ldots$,

$$
K_{t}^{(\rho)}(\nu)=\sum_{i \geqslant p} c_{i} \lambda_{i}^{\nu} S_{i}^{(\rho)}(0) \quad \text { and } \quad K_{t}^{(\rho)}(-\nu)=\sum_{i \leqslant q} c_{i} \lambda_{i}^{-\nu} S_{i}^{(\rho)}(0)
$$

So $K_{t}^{(\rho)}(\nu)=c_{p} \lambda_{p}^{\nu} S_{p}^{(\rho)}(0)+O\left(\lambda_{p}^{\nu}\right)$ as $\nu \rightarrow \infty$ and $K_{t}^{(\rho)}(-\nu)=c_{q} \lambda_{q}^{-\nu} S_{q}^{(\rho)}(0)+O\left(\nu_{q}^{-\nu}\right)$ as $\nu \rightarrow \infty$. Thus for large enough $N$,

$$
S\left(K_{t}^{\prime}(N), \ldots, K_{t}^{(n-r)}(N)\right)=S\left(S_{p}^{\prime}(0), \ldots, S_{p}^{(n-r)}(0)\right)=p-1
$$

and

$$
S\left(K_{t}^{\prime}(-N), \ldots, K_{t}^{(n-r)}(-N)\right)=S\left(S_{q}^{\prime}(0), \ldots, S_{q}^{(n-r)}(0)\right)=q-1 .
$$

We now show that $K_{t}$ is oscillating in $(-N, N)$. For suppose $K_{t}=0$ on some interval $(a, b) \in(-N, N)$. Since $K_{t} \in C^{n-r}(\mathbf{R})$ and is a piecewise polynomial with 
knots at $\mathbf{Z} \cup\{t\}, a, b \in \mathbf{Z} \cup\{t\}$. Furthermore, either $a<t$ or $b>t$. Suppose $a<t$. Then $K_{t}^{(\rho)}(a)=0, \rho=0, \ldots, n-r$, and $K_{t}\left(a-1+x_{i}\right)=0, i=0, \ldots, r-1$, implies that $K_{t}=0$ on $(a-1, a)$. Hence $K_{t}^{(\rho)}(a-1)=0, \rho=0, \ldots, n-r$. By induction we have $K_{t}^{(\rho)}(-N)=0, \rho=0, \ldots, n-r$, which contradicts (2.9). Similarly $b>t$ leads to $K_{t}^{(\rho)}(N)=0, \rho=0, \ldots, n-r$, which contradicts (2.8).

Now $K_{t}$ has exact degree $n$ in $(0,1)$ and so we may apply Theorem 2.1 of [5] to $g:=\left.K_{t}\right|_{(-N, N)}$ to give

$$
\begin{aligned}
Z(g) \leqslant & (2 N-1) r+1+S^{-}\left(g\left(-N^{+}\right), \ldots, g^{(n)}\left(-N^{+}\right)\right) \\
& -S^{+}\left(g\left(N^{-}\right), \ldots, g^{(n)}\left(N^{-}\right)\right) \\
\leqslant & (2 N-1) r+1+(q-1+r)-p \quad\left(\text { since } g\left(-N^{+}\right)=g\left(N^{-}\right)=0\right) \\
= & 2 N r+q-p \leqslant 2 N r-1 .
\end{aligned}
$$

But $K_{t}$ has $2 N r-1$ zeros in $(-N, N)$ at points as in (2.5) and hence these are the only zeros of $K_{t}$ in $(-N, N)$. Since $N$ can be arbitrarily large, (a) follows.

We must also consider the special cases $r=n$ or $n+1$, for which it is easy to see the following. If $t \in(\nu, \nu+1)$, then $K_{t}$ vanishes outside $[\nu, \nu+1]$ and vanishes in $(\nu, \nu+1)$ only at $\nu+x_{i}, i=1, \ldots, r$. Similarly if $x \in(\nu, \nu+1)$, then $\tilde{K}_{x}$ vanishes outside $[\nu, \nu+1]$ and vanishes nowhere in $(\nu, \nu+1)$.

THEOREM 2.1. If $f \in \mathscr{F}_{n, r}$ and $f^{(n+1-r)}$ is of power growth, then

$$
f(x)-S_{n, r}(f ; x)=\int_{-\infty}^{\infty} K(x, t) f^{(n+1)}(t) d t .
$$

Proof. By (2.4) we may assume $0<x \leqslant 1$. We may also assume $x \neq x_{i}, i=$ $1, \ldots, r$, since otherwise $(2.10)$ is trivially satisfied.

Now integrating by parts and applying (2.6) gives, for any $\nu \in \mathbf{Z}$,

$$
\int_{\nu}^{\nu+1} K(x, t) f^{(n+1)}(t) d t=(-1)^{r} \int_{\nu}^{\nu+1} \tilde{K}_{x}^{(r)}(t) f^{(n+1-r)}(t) d t .
$$

So $\int_{-\infty}^{\infty} K(x, t) f^{(n+1)}(t) d t=(-1)^{r} \int_{-\infty}^{\infty} \tilde{K}_{x}^{(r)}(t) f^{(n+1-r)}(t) d t$ which converges since $f^{(n+1-r)}$ is of power growth and $K_{x}^{(r)}$ decays exponentially. Also $f^{(\rho)}$ is of power growth for $0 \leqslant \rho \leqslant n+1-r$ and so we may integrate by parts to give

$$
(-1)^{r} \int_{-\infty}^{\infty} \tilde{K}_{x}^{(r)}(t) f^{(n+1-r)}(t) d t=(-1)^{n} \int_{-\infty}^{\infty} \tilde{K}_{x}^{(n)}(t) f^{\prime}(t) d t .
$$

Now $(-1)^{n} \tilde{K}_{x}^{(n)}(t)=(x-t)_{+}^{0}-\sum_{i=1}^{r} \sum_{\nu=-\infty}^{\infty}\left(\nu+x_{i}-t\right)_{+}^{0} L_{i}(x-\nu)$ and so

$$
\begin{aligned}
(-1)^{n} \int_{-\infty}^{\infty} \tilde{K}_{x}^{(n)}(t) f^{\prime}(t) d t & =f(x)-\sum_{i=1}^{r} \sum_{\nu=-\infty}^{\infty} f\left(\nu+x_{i}\right) L_{i}(x-\nu) \\
& =f(x)-S_{n, r}(f ; x) .
\end{aligned}
$$

3. Proof of Theorem 1. We see immediately from Theorem 2.1 that for $f \in \mathscr{F}_{n, r}$ with $f^{(n+1-r)}$ of power growth and $\left\|f^{(n+1)}\right\|_{\infty}<\infty$

$$
\left\|f-S_{n, r}(f ;)\right\|_{\infty} \leqslant \sup _{x \in \mathbf{R}}\left\{\int_{-\infty}^{\infty}|K(x, t)| d t\right\}\left\|f^{(n+1)}\right\|_{\infty} .
$$


Now there is an eigenspline $E_{n+1, r} \in \mathcal{S}_{n+1, r}$ with eigenvalue $(-1)^{r}$ which vanishes at $\nu+x_{i}, \forall \nu \in \mathbf{Z}$ and $i=1, \ldots, r$. We assume it is normalised so that $\left|E_{n+1, r}^{(n+1)}\right|=1$.

Putting $f=E_{n+1, r}$ in (2.10), we see from Lemma 2.1(b) that

$$
\left|E_{n+1, r}(x)\right|=\int_{-\infty}^{\infty}|K(x, t)| d t, \quad \forall x \in \mathbf{R},
$$

so that the constant $K$ in (1.6) is given by

$$
K=\left\|E_{n+1, r}\right\|_{\infty} \text {. }
$$

Following the definitions of Cavaretta [1] and Goodman [4], we let $\mathcal{E}_{n+1, r} \in \mathcal{S}_{n+1, r}$ denote the Euler-Chebyshev spline, normalised so that $\left|\mathcal{E}_{n+1, r}^{(n+1)}\right|=1$. Now the zeros of $\varepsilon_{n+1, r}$ are points $\nu+\beta_{i}, \nu \in \mathbf{Z}$, where $\beta_{i}, i=1, \ldots, r$, are symmetric about $x=\frac{1}{2}$, and $0<\beta_{1}<\cdots<\beta_{r} \leqslant 1$, with equality iff $n+r$ is even. Furthermore

$$
\mathcal{E}_{n+1, r}(x+1)=(-1)^{r} \mathcal{E}_{n+1, r}(x), \quad \forall x \in \mathbf{R},
$$

and $f=\mathcal{E}_{n+1, r}$ minimises $\|f\|_{\infty}$ over all $f \in \mathcal{S}_{n+1, r}$ with $\left\|f^{(n+1)}\right\|_{\infty}=1$. The result follows.

4. Convergence of $S_{n, r}(f ;)$. Henceforth we shall examine the behaviour of $S_{n, r}(f, x)$ as $n \rightarrow \infty$. Analogous problems were studied in [6, 9 and 10]. We first derive an estimate for $\left\|E_{n+1, r}\right\|_{\infty}$.

LeMma 4.1. For fixed $\mathbf{x}$ and $r \geqslant 1, \exists K_{r}$ such that

$$
\left\|E_{n+1, r}\right\|_{\infty} \leqslant K_{r} /(r \pi)^{n+1}, \quad \forall n \geqslant r-1 .
$$

Proof. First, assume that $r$ is odd. For $x \in[0,1]$, let

$$
E_{n+1, r}(x)=a_{0} E_{n+1}(x)+a_{1} E_{n}(x)+\cdots+a_{n} E_{1}(x)+a_{n+1} E_{0}(x),
$$

where $E_{k}(x), k=0,1, \ldots, n+1$, are the Euler polynomials. The conditions $E_{n+1, r}^{(\rho)}(1)=-E_{n+1, r}^{(\rho)}(0)$ for $\rho=0,1, \ldots, n-r+1$ imply that $a_{n-k+1}=0 \forall k=$ $0,1, \ldots, n-r+1$. Hence for $x \in[0,1]$,

$$
E_{n+1, r}(x)=a_{0} E_{n+1}(x)+a_{1} E_{n}(x)+\cdots+a_{r-1} E_{n-r+2}(x) .
$$

Now $E_{n+1, r}\left(x_{i}\right)=0, i=1,2, \ldots, r$, gives a homogeneous system of equations $a_{0} E_{n+1}\left(x_{i}\right)+a_{1} E_{n}\left(x_{i}\right)+\cdots+a_{r-1} E_{n-r+2}\left(x_{i}\right)=0, i=1,2, \ldots, r$, whose determinant must be zero. Hence we can write

$$
E_{n+1, r}(x)=\frac{\operatorname{det}\left(E_{n-m+2}\left(\beta_{l}\right)\right)_{l, m=1}^{r}}{\operatorname{det}\left(E_{n-m+2}\left(x_{l}\right)\right)_{l, m=2}^{r}} \quad \forall x \in[0,1]
$$

where

$$
\beta_{l}= \begin{cases}x & \text { if } l=1 \\ x_{l} & \text { if } l \neq 1\end{cases}
$$


Using the Fourier expansions of the Euler polynomials $E_{k}(x)$ we obtain

$$
\begin{aligned}
\left|E_{n+1, r}(x)\right| & =\frac{2}{\pi^{n+2}}\left|\frac{\operatorname{det}\left(\sum_{-\infty}^{\infty} e^{2 k \pi i \beta_{l}} /(2 k+1)^{n-m+3}\right)_{l, m=1}^{r}}{\operatorname{det}\left(\sum_{-\infty}^{\infty} e^{2 k \pi i x_{1}} /(2 k+1)^{n-m+3}\right)_{l, m=2}^{r}}\right| \\
& =\left(\frac{2^{r}}{\pi^{n+2}}\right)\left|\frac{\sum_{k_{1}, k_{2}, \ldots, k_{r}} V\left(k_{1}, k_{2}, \ldots, k_{r}\right) \prod_{j=1}^{r} e^{2 k_{j} \pi i \beta_{l}} /\left(2 k_{j}+1\right)^{n+1}}{\sum_{k_{2}, k_{3}, \ldots, k_{r}} V\left(k_{2}, k_{3}, \ldots, k_{r}\right) \prod_{j=2}^{r} e^{2 k_{j} \pi i x_{j}} /\left(2 k_{j}+1\right)^{n+1}}\right|
\end{aligned}
$$

where $V\left(a_{1}, a_{2}, \ldots, a_{r}\right)$ denotes the Vandermonde determinant $\operatorname{det}\left(a_{m}^{l-1}\right)_{l, m=1}^{r}=$ $\prod_{1 \leqslant j<k \leqslant r}\left(a_{k}-a_{j}\right)$. A straightforward computation gives

$$
\left|E_{n+1, r}(x)\right|
$$

$$
\left(\frac{2^{r}}{\pi^{n+2}}\right)\left|\frac{\sum_{k_{1}<\cdots<k_{r}} V\left(k_{1}, k_{2}, \ldots, k_{r}\right) \operatorname{det}\left(e^{2 k_{m} \pi i \beta_{l}}\right)_{l, m=1}^{r} \sum_{j=1}^{r}\left(2 k_{j}+1\right)^{-(n+2)}}{\sum_{k_{2}<\cdots<k_{r}} V\left(k_{2}, k_{3}, \ldots, k_{r}\right) \operatorname{det}\left(e^{2 k_{m} \pi i x_{l}}\right)_{l, m=2}^{r} \sum_{j=2}^{r}\left(2 k_{j}+1\right)^{-(n+1)}}\right| .
$$

The dominant term in the numerator of (4.2) is

$$
\begin{aligned}
\prod_{j=1}^{r}(2 j-r)^{-(n+2)} & \left\{V\left(-\frac{(r-1)}{2},-\frac{(r-3)}{2}, \ldots, \frac{r-1}{2}\right)\left(e^{(2 m-r-1) \pi i \beta_{l}}\right)_{l, m=1}^{r}\right. \\
& \left.-V\left(-\frac{(r+1)}{2},-\frac{(r-1)}{2}, \ldots, \frac{r-3}{2}\right)\left(e^{(2 m-r-3) \pi i \beta_{l}}\right)_{l, m=1}^{r}\right\} .
\end{aligned}
$$

The dominant term in the denominator is

$$
\begin{aligned}
\prod_{j=2}^{r}(2 j-r-2)^{-(n+1)} V\left(-\frac{(r-1)}{2},-\frac{(r-3)}{2}, \ldots, \frac{(r-3)}{2}\right) \\
\times \operatorname{det}\left(e^{(2 m-r-3) \pi i x_{l}}\right)_{l, m=2 .}^{r} .
\end{aligned}
$$

It follows from (4.2), (4.3) and (4.4) that $\forall x \in[0,1]$

$$
\left|E_{n+1, r}(x)\right|=\left(\frac{2^{r}}{\pi^{n+2}}\right)\left|\frac{\Pi_{j=2}^{r}(2 j-r-2)^{n+1}}{\prod_{j=1}^{r}(2 j-r)^{n+2}}\right| O(1),
$$

and (4.1) follows for odd $r$.

If $r$ is even, a similar argument shows that for $x \in[0,1]$ the eigensplines $E_{n+1, r}(x)$ may be expressed in terms of Bernoulli polynomials $B_{k}(x)$ as follows:

$$
E_{n+1, r}(x)=\frac{\operatorname{det}^{*}\left(B_{n-m+3}\left(\beta_{l}\right)\right)_{l=1: m=2}^{r}}{\operatorname{det}^{*}\left(B_{n-m+3}\left(x_{l}\right)\right)_{l=2 ; m=3}^{r}},
$$


where det* means that all the entries in the last row of the determinant are 1. Expanding each determinant along the last row and applying a similar method to each term gives the inequality (4.1) for $r$ even.

Now recall the definition of $B_{\sigma}$ in $\S 1$. We shall need Bernstein's theorem that if $f \in B_{\sigma}$ then, for each integer $n, f^{(n)} \in B_{\sigma}$ and

$$
\left\|f^{(n)}\right\|_{\infty} \leqslant \sigma^{n}\|f\|_{\infty} .
$$

From (1.6), (3.1), (4.1) and (4.5), we can immediately deduce Theorem 2.

COROLlaRY 4.1. If $f \in B_{\sigma}$ and $\sigma<r \pi$, then

$$
\lim _{n \rightarrow \infty} S_{n, r}(f ; x)=f(x) \text { uniformly on } \mathbf{R} \text {. }
$$

COROLLARY 4.2. If $f \in B_{r \pi}$, then for all $n \geqslant r-1$

$$
\left\|f-S_{n, r}(f)\right\|_{\infty} \leqslant K_{r}\|f\|_{\infty} \text {. }
$$

We now follow a similar approach to that of Schoenberg [10] in proving Theorem 3. First we introduce the class $B_{r \pi}^{*}$ of functions which are uniform limits on $\mathbf{R}$ of functions belonging to $B_{p}$ for $p<r \pi$, i.e. $f \in B_{r \pi}^{*}$ if and only if $\exists f_{j} \in B_{p}, p_{j}<r \pi$, $j=1,2,3, \ldots$, such that $\left\|f-f_{j}\right\|_{\infty} \rightarrow 0$ as $j \rightarrow \infty$.

LEMMA 4.2. If $f \in B_{r \pi}^{*}$ then

$$
\lim _{n \rightarrow \infty} S_{n, r}(f ; x)=f(x) \text { uniformly. }
$$

Proof. Suppose $f_{j} \in B_{p_{j}}, p_{j}<r \pi, j=1,2,3, \ldots$, and $\left\|f-f_{j}\right\|_{\infty} \rightarrow 0$ as $j \rightarrow \infty$. Then $f-S_{n, r}(f ;)=f-f_{j}-S_{n, r}\left(f-f_{j} ;\right)+f_{j}-S_{n, r}\left(f_{j} ;\right)$ and using Corollaries 4.1 and 4.2, the result follows as in [10].

Before we prove Theorems 3 and 4 we first study the behaviour of the spline functions $S_{n, r}(\cos r \pi x)$ and $S_{n, r}(\sin r \pi x)$ that interpolate $\cos r \pi x$ and $\sin r \pi x$ respectively at $\nu+x_{i}, i=1,2, \ldots, r, \nu \in \mathbf{Z}$. In order to simplify writing, we define $\alpha_{1}, \ldots, \alpha_{r}$ by

$$
\begin{gathered}
\alpha_{i}=x_{i}, \quad i=1, \ldots, r, \text { if } n+r \text { is odd, } \\
\alpha_{1}=0 \text { and } \alpha_{i}=x_{i-1}, \quad i=1, \ldots, r-1, \text { if } n+r \text { is even. }
\end{gathered}
$$

We first introduce the exponential Euler splines

$$
S_{n, r}(x ; u)=\sum_{s=1}^{r} e^{i u x} \Omega_{s}(x, u) \quad \forall x \in \mathbf{R},
$$

where

$$
\Omega_{s}(x, u)=\frac{\operatorname{det}\left(\sum_{k=-\infty}^{\infty} e^{2 k \pi i \beta_{l}} /(u+2 k \pi)^{n-m+2}\right)_{l, m=1}^{r}}{\operatorname{det}\left(\sum_{k=-\infty}^{\infty} e^{2 k \pi i \alpha_{l}} /(u+2 k \pi)^{n-m+2}\right)_{l, m=1}^{r}},
$$

$(r-2) \pi<u \leqslant r \pi$, and

$$
\beta_{l}= \begin{cases}x & \text { if } l=s \\ \alpha_{l} & \text { if } l \neq s\end{cases}
$$


Clearly $S_{n, r}\left(\nu+\alpha_{i} ; u\right)=e^{i u\left(\nu+\alpha_{1}\right)} \quad \forall i=1,2, \ldots, r, \quad \nu \in \mathbf{Z}$, and $S_{n, r}(x, r \pi)=$ $S_{n, r}(\cos r \pi x)+i S_{n, r}(\sin r \pi x)$. Therefore we are interested in the limit of $S_{n, r}(x ; r \pi)$ as $n \rightarrow \infty$. First we prove

LEMma 4.3. For $s=1,2, \ldots, r$,

$$
\lim _{n \rightarrow \infty} \Omega_{s}(x, r \pi)=e^{\pi i\left(\alpha_{1}-x\right)} \cos \pi\left(\alpha_{s}-x\right) \frac{V\left(e^{-2 \pi i \beta_{1}}, e^{-2 \pi i \beta_{2}}, \ldots, e^{-2 \pi i \beta_{r}}\right)}{V\left(e^{-2 \pi i \alpha_{1}}, e^{-2 \pi i \alpha_{2}}, \ldots, e^{-2 \pi i \alpha_{r}}\right)}
$$

uniformly on $\mathbf{R}$.

Proof. Assume $(r-1) \pi<u<r \pi$. A straightforward computation shows that

$$
\begin{aligned}
& \Omega_{s}(x, u)=\frac{\sum_{k_{1}, k_{2} \ldots k_{r}} V\left(k_{1}, k_{2}, \ldots, k_{r}\right) \prod_{j=1}^{r} e^{2 k_{,} \pi i \beta_{l}} /\left(u+2 k_{j} \pi\right)^{n+1}}{\sum_{k_{1}, k_{2}, \ldots, k_{r}} V\left(k_{1}, k_{2}, \ldots, k_{r}\right) \prod_{j=1}^{r} e^{2 k_{,} \pi i \alpha_{l}} /\left(u+2 k_{j} \pi\right)^{n+1}} \\
& =\frac{\sum_{k_{1}<\cdots<k_{r}} V\left(k_{1}, k_{2}, \ldots, k_{r}\right) \operatorname{det}\left(e^{2 k_{m} \pi i \beta_{l}}\right)_{l, m=1}^{r} \prod_{j=1}^{r}\left(u+2 k_{j} \pi\right)^{-n-1}}{\sum_{k_{1}<\cdots<k_{r}} V\left(k_{1}, k_{2}, \ldots, k_{r}\right) \operatorname{det}\left(e^{2 k_{m} \pi i \alpha_{l}}\right)_{l, m=1}^{r} \prod_{j=1}^{r}\left(u+2 k_{j} \pi\right)^{-n-1}} \\
& =\frac{\operatorname{det}\left(e^{-2(m-1) \pi i \beta_{l}}\right)_{l, m=1}^{r}+\left(\frac{u}{u-2 r \pi}\right)^{n+1} \operatorname{det}\left(e^{-2 m \pi i \beta_{l}}\right)_{l, m=1}^{r}+O\left(\left|\frac{u-2 \pi}{u-2 r \pi}\right|^{n+1}\right)}{\operatorname{det}\left(e^{-2(m-1) \pi i \alpha_{l}}\right)_{l, m=1}^{r}+\left(\frac{u}{u-2 r \pi}\right)^{n+1} \operatorname{det}\left(e^{-2 m \pi i \alpha_{l}}\right)_{l, m=1}^{r}+O\left(\left|\frac{u-2 \pi}{u-2 r \pi}\right|^{n+1}\right)} .
\end{aligned}
$$

Taking the limit as $u \rightarrow r \pi$, after some simplification, we obtain

$$
\Omega_{s}(x, u)=\frac{V\left(e^{-2 \pi i \beta_{1}}, \ldots, e^{-2 \pi i \beta_{r}}\right)\left(1+(-1)^{n+1} e^{-2 \pi i \Sigma_{l}^{r}, \beta_{l}}\right)+O\left((r-2 / r)^{n+1}\right)}{V\left(e^{-2 \pi i \alpha_{1}}, \ldots, e^{-2 \pi i \alpha_{r}}\right)\left(1+(-1)^{n+1} e^{-2 \pi i \Sigma_{l=1}^{r} \alpha_{l}}\right)+O\left((r-2 / r)^{n+1}\right)} .
$$

Now suppose $n$ even. If $r$ is even, $\alpha_{1}=0$ and

$$
\sum_{l=1}^{r} \alpha_{l}=\frac{r-2}{2}+\frac{1}{2}
$$

If $r$ is odd, $\alpha_{1}>0$ and

$$
\sum_{l=1}^{r} \alpha_{l}=\frac{r-1}{2}+\frac{1}{2}
$$

The result (4.7) then follows from (4.8), (4.9) and (4.10). For odd $n$,

$$
\sum_{l=1}^{r} \alpha_{l}= \begin{cases}(r-1) / 2 & \text { if } r \text { is odd } \\ r / 2 & \text { if } r \text { is even, }\end{cases}
$$

and (4.7) follows similarly. 
LEMMA 4.4. If $\alpha_{1}=0$, the following limits hold uniformly:

$$
\lim _{n \rightarrow \infty} S_{n, r}(\cos r \pi x)=\cos r \pi x
$$

$$
\lim _{n \rightarrow \infty} S_{n, r}(\sin r \pi x)=\sin r \pi x+(-1)^{r} 2^{r-1} \prod_{i=1}^{r} \sin \pi\left(x-\alpha_{i}\right) .
$$

If $\alpha_{1}>0$, the following limits hold uniformly:

$$
\begin{gathered}
\lim _{n \rightarrow \infty} S_{n, r}(\cos r \pi x)=\cos r \pi x+(-1)^{r-1} 2^{r-1} \prod_{i=1}^{r} \sin \pi\left(x-\alpha_{i}\right) \\
\lim _{n \rightarrow \infty} S_{n, r}(\sin r \pi x)=\sin r \pi x
\end{gathered}
$$

Proof. First we write

$$
\begin{aligned}
& \frac{V\left(e^{-2 \pi i \beta_{1}}, e^{-2 \pi i \beta_{2}}, \ldots, e^{-2 \pi i \beta_{r}}\right)}{V\left(e^{-2 \pi i \alpha_{1}}, e^{-2 \pi i \alpha_{2}}, \ldots, e^{-2 \pi i \alpha_{r}}\right)}=\frac{\prod_{1 \leqslant l<k \leqslant r}\left(e^{-2 \pi i \beta_{k}}-e^{-2 \pi i \beta_{l}}\right)}{\prod_{1 \leqslant l<k \leqslant r}\left(e^{-2 \pi i \alpha_{k}}-e^{-2 \pi i \alpha_{l}}\right)} \\
&=e^{(r-1) \pi i\left(\alpha_{s}-x\right)} \frac{\prod_{\substack{1 \leqslant k \leqslant r \\
\prod_{1 \leqslant l<k \leqslant r} \sin \pi\left(\beta_{k}-\beta_{l}\right)}} \sin \pi\left(\alpha_{k}-\alpha_{l}\right)}{\prod_{k=1}^{r} \sin \pi\left(x-\alpha_{k}\right)} \\
&=e^{(r-1) \pi i\left(\alpha_{s}-x\right)}, \\
& \prod_{k=1}^{r} \sin \pi\left(\alpha_{s}-\alpha_{k}\right)
\end{aligned}
$$

where $\Pi_{k=1}^{\prime r}$ indicates that the factor involving $k=s$ is omitted. Hence it follows from (4.6) and (4.7) that

$$
\begin{aligned}
\lim _{n \rightarrow \infty} S_{n, r}(x, r \pi) & =\sum_{s=1}^{r} e^{r \pi i \alpha_{s}} \cos \pi\left(x-\alpha_{s}\right) \frac{\prod_{k=1}^{r} \sin \pi\left(x-\alpha_{k}\right)}{\prod_{k=1}^{r} \sin \pi\left(\alpha_{s}-\alpha_{k}\right)} . \\
& \equiv \phi(x)+i \psi(x),
\end{aligned}
$$

where

$$
\phi(x)=\sum_{s=1}^{r} \cos r \pi \alpha_{s} \cos \pi\left(x-\alpha_{s}\right) \frac{\prod_{k=1}^{r} \sin \pi\left(x-\alpha_{k}\right)}{\prod_{k=1}^{r} \sin \pi\left(\alpha_{s}-\alpha_{k}\right)}
$$


and

$$
\psi(x)=\sum_{s=1}^{r} \sin r \pi \alpha_{s} \cos \pi\left(x-\alpha_{s}\right) \frac{\prod_{k=1}^{r} \sin \pi\left(x-\alpha_{k}\right)}{\prod_{k=1}^{r} \sin \pi\left(\alpha_{s}-\alpha_{k}\right)} .
$$

Clearly $\lim _{n \rightarrow \infty} S_{n, r}(\cos r \pi x)=\phi(x), \lim _{n \rightarrow \infty} S_{n, r}(\sin r \pi x)=\psi(x)$.

Next, we want to simplify $\phi(x)$ and $\psi(x)$. First we consider $r$ even. The Gauss trigonometric interpolation formula gives

$$
\phi(x)=\cos r \pi x+\lambda \prod_{k=1}^{r} \sin \pi\left(x-\alpha_{k}\right) .
$$

To determine $\lambda$, we write

$$
\begin{aligned}
& \cos \pi\left(\alpha_{s}-x\right) \prod_{k=1}^{r} \sin \pi\left(x-\alpha_{k}\right)=\frac{1}{2(2 i)^{r-1}}\left(e^{\pi i\left(x-\alpha_{s}\right)}+e^{-\pi i\left(x-\alpha_{s}\right)}\right) \\
& \cdot\left(\exp \left[\pi i\left((r-1) x-\sum_{k=1}^{r} \alpha_{k}\right)\right]+\cdots+(-1)^{r-1} \exp \left[-\pi i\left((r-1) x-\sum_{k=1}^{r} \alpha_{k}\right)\right]\right)
\end{aligned}
$$

and

$$
\begin{aligned}
\prod_{k=1}^{r} \sin \pi\left(x-\alpha_{k}\right)=\frac{1}{(2 i)^{r}}(\exp [\pi i(r x & \left.\left.-\sum_{k=1}^{r} \alpha_{k}\right)\right]+\cdots \\
& \left.+(-1)^{r} \exp \left[-\pi i\left(r x-\sum_{k=1}^{r} \alpha_{k}\right)\right]\right) .
\end{aligned}
$$

Equating the highest order terms in (4.15), it follows from (4.15)-(4.17) that

$$
\begin{aligned}
\frac{1}{2(2 i)^{r-1}} \sum_{s=1}^{r} A_{s}\left\{\cos \left(\pi\left(r x-\sum_{k=1}^{r} \alpha_{k}\right)\right)+i \sin \left(\pi\left(r x-\sum_{k=1}^{r} \alpha_{k}\right)\right)\right. \\
\left.+(-1)^{r-1}\left[\cos \left(\pi\left(r x-\sum_{k=1}^{r} \alpha_{k}\right)\right)-i \sin \left(\pi\left(r x-\sum_{k=1}^{r} \alpha_{k}\right)\right)\right]\right\} \\
=\cos r \pi x+\lambda\left\{\cos \pi\left(r x-\sum_{k=1}^{r} \alpha_{k}\right)+i \sin \pi\left(r x-\sum_{k=1}^{r} \alpha_{k}\right)\right. \\
\left.+(-1)^{r}\left[\cos \pi\left(r x-\sum_{k=1}^{r} \alpha_{k}\right)-i \sin \pi\left(r x-\sum_{k=1}^{r} \alpha_{k}\right)\right]\right\},
\end{aligned}
$$

where $A_{s}=\cos \pi r \alpha_{s} / \Pi_{k=1}^{\prime r} \sin \pi\left(\alpha_{s}-\alpha_{k}\right)$. 
Since $r$ is even it follows that

$$
\frac{-2}{(2 i)^{r}} \sum_{s=1}^{r} A_{s} \sin \pi\left(r x-\sum_{k=1}^{r} \alpha_{k}\right)=\cos r \pi x+\frac{2 \lambda \cos \pi\left(r x-\sum_{k=1}^{r} \alpha_{k}\right)}{(2 i)^{r}}
$$

Now if $\alpha_{1}=0$, then $\sum_{k=1}^{r} \alpha_{k}=r / 2-\frac{1}{2}$, so that (4.18) becomes

$$
\frac{(-1)^{(r+2) / 2}}{(2 i)^{r}} \cos r \pi x\left(\sum_{s=1}^{r} A_{s}\right)=\cos \pi r x+\frac{(-1)^{(r+2) / 2} 2 \lambda \sin r \pi x}{(2 i)^{r}} \text {. }
$$

Hence $\lambda=0$. This proves (4.11) for $r$ even.

If $\alpha_{1} \neq 0$, then $\sum_{k=1}^{r} \alpha_{k}=r / 2$, so that (4.18) becomes

$$
\frac{-2}{2^{r}} \sin r \pi x\left(\sum_{s=1}^{r} A_{s}\right)=\cos \pi r x+\frac{2 \lambda \cos \pi r x}{2^{r}} \text {. }
$$

Hence $\lambda=-2^{r-1}$. This proves (4.13) for $r$ even. The proof of (4.12) and (4.14) for $r$ even are the same.

Next we consider $r$ odd. If $\alpha_{1} \neq 0$, we let $\alpha_{0}=0$ and write

$$
\phi(x)=\frac{1}{\sin \pi x} \sum_{s=0}^{r} \cos r \pi \alpha_{s} \cos \pi\left(x-\alpha_{s}\right) \frac{\prod_{k=0}^{r} \sin \pi\left(x-\alpha_{k}\right)}{\prod_{k=0}^{r} \sin \pi\left(\alpha_{s}-\alpha_{k}\right)} .
$$

The Gauss interpolation formula again gives

$$
\begin{array}{r}
\sum_{s=0}^{r} \cos r \pi \alpha_{s} \sin \pi \alpha_{s} \cos \pi\left(x-\alpha_{s}\right) \frac{\prod_{k=0}^{r} \sin \pi\left(x-\alpha_{k}\right)}{\prod_{k=0}^{r} \sin \left(\alpha_{s}-\alpha_{k}\right)} \\
=\cos r \pi x \sin \pi x+\lambda \prod_{k=0}^{r} \sin \pi\left(x-\alpha_{k}\right) .
\end{array}
$$

A similar calculation gives $\lambda=2^{r-1}$, so that (4.19) gives $\phi(x)=\cos r \pi x+$ $2^{r-1} \prod_{k=1}^{r} \sin \pi\left(x-\alpha_{k}\right)$. This proves (4.13) for $r$ odd. The proof of (4.14) is similar.

If $\alpha_{1}=0$, we let $\alpha_{k+1}=\frac{1}{2}$ and write

$$
\phi(x)=\frac{1}{\cos \pi x} \sum_{s=1}^{r+1} \cos r \pi \alpha_{s} \cos \pi \alpha_{s} \cos \pi\left(x-\alpha_{s}\right) \frac{\prod_{k=1}^{r+1} \sin \pi\left(x-\alpha_{k}\right)}{\prod_{k=1}^{r+1} \sin \pi\left(\alpha_{s}-\alpha_{k}\right)}
$$

and (4.11) and (4.12) for odd $r$ are proved similarly.

Proof of Theorem 3. Let

$$
\alpha_{0}(u)= \begin{cases}\alpha(-r \pi+0) & \text { if } u=-r \pi \\ \alpha(u) & \text { if }-r \pi<u<r \pi \\ \alpha(r \pi-0) & \text { if } u=r \pi\end{cases}
$$


Then $\alpha_{0}(u)$ has no jumps at $\pm r \pi$. Define

$$
f_{0}(x)=\int_{-r \pi}^{r \pi} e^{i u x} d \alpha_{0}(u) \quad \forall x \in \mathbf{R} .
$$

Setting $A_{1}=\alpha(-r \pi+0)-\alpha(-r \pi), A_{2}=\alpha(r \pi)-\alpha(r \pi-0)$, we can write $f(x)=$ $f_{0}(x)+A_{1} e^{-r \pi i x}+A_{2} e^{r \pi i x}$, and setting $A=A_{1}+A_{2}, B=i\left(A_{2}-A_{1}\right)$ we obtain

$$
f(x)=f_{0}(x)+A \cos r \pi x+B \sin r \pi x \quad \forall x \in \mathbf{R} .
$$

Now $f_{0} \in B_{r \pi}^{*}$ since $f_{0}$ is the uniform limit of the sequence $\left\{f_{j}\right\}, f_{j} \in B_{p_{j}}$, defined by $f_{j}(x)=\int_{-p_{j}}^{p_{j}} e^{i u x} d \alpha_{0}(u)$, with $0<p_{j}<r \pi, p_{j} \rightarrow r \pi$ as $j \rightarrow \infty$. By Lemma 4.2 we conclude that $\lim _{n \rightarrow \infty} S_{n, r}\left(f_{0} ; x\right)=f_{0}(x)$ uniformly on $\mathbf{R}$. The theorem now follows from (4.21) and Lemma 4.4.

Finally we consider the class $\leftrightarrow \uparrow$ of almost periodic functions in the sense of Bohr. To every $f \in \mathbb{Q} \mathscr{P}$ corresponds a Fourier series

$$
f(x) \sim \sum_{\nu=1}^{\infty} A_{\nu} e^{i \lambda_{\nu}, x},
$$

where $\lambda_{\nu}$ are real numbers, called the Fourier exponents of $f$. Also for $\sigma \geqslant 0$,

$$
\mathbb{P} \cap B_{\sigma}=\left\{f: f \in \mathbb{Q} \oplus,-\sigma \leqslant \lambda_{\nu} \leqslant \sigma\right\} .
$$

Proof of Theorem 4. Suppose $f \in \mathcal{Q} \mathscr{P} \cap B_{r \pi}$. Then its Fourier exponents $\lambda_{\nu}$, $\nu=1,2,3, \ldots$, satisfy $-r \pi \leqslant \lambda_{\nu} \leqslant r \pi$.

Without loss of generality we may assume that $\lambda_{1}=-r \pi, \lambda_{2}=r \pi$ with the understanding that $A_{1}=0$ if the exponent $-r \pi$ is absent, and similarly that $A_{2}=0$ if exponent $r \pi$ is absent.

Let

$$
A_{1} e^{-r \pi i x}+A_{2} e^{r \pi i x}=A \cos r \pi x+B \sin r \pi x,
$$

where $A=A_{2}+A_{1}, B=i\left(A_{2}-A_{1}\right)$. It follows that the function

$$
g(x)=f(x)-A \cos r \pi x-B \sin r \pi x
$$

has Fourier series $g(x) \sim \sum_{\nu=3}^{\infty} A_{\nu} e^{i \lambda_{\nu} x}$ where $-r \pi<\lambda_{\nu}<r \pi \forall \lambda=3,4,5, \ldots$ A similar argument as in [10] shows that $g \in B_{r \pi}^{*}$. It follows from Lemma 4.2 that

$$
S_{n, r}(g ; x) \rightarrow g(x) \text { uniformly on } \mathbf{R} \text {. }
$$

The theorem then follows from (4.22), (4.23) and Lemma 4.4.

\section{REFERENCES}

1. A. S. Cavaretta, Jr., On cardinal perfect splines of least sup-norm on the real axis, J. Approx. Theory 8 (1973), 285-303.

2. Carl de Boor and I. J. Schoenberg, Cardinal interpolation and spline functions. VIII. The Budan-Fourier theorem for splines and applications, Spline Functions (Proc. Internat. Sympos., Karlsruhe, 1975), Lecture Notes in Math., vol. 501, Springer-Verlag, Berlin and New York, 1976, pp. 1-79.

3. P. J. Davis, Interpolation and approximation, Blaisdell, Waltham, Mass., 1963.

4. T. N. T. Goodman, Necessary conditions for the convergence of cardinal Hermite splines as their degree tends to infinity, Trans. Amer. Math. Soc. 255 (1979), 231-241.

5. T. N. T. Goodman and S. L. Lee, The Budan-Fourier theorem and Hermite-Birkhoff spline interpolation, Trans. Amer. Math. Soc. 271 (1982), 451-467.

6. M. J. Marsden and S. D. Riemenschneider, Cardinal Hermite spline interpolation: convergence as the degree tends to infinity, Trans. Amer. Math. Soc. 235 (1976), 221-244. 
7. C. A. Micchelli, Oscillation matrices and cardinal spline interpolation, Studies in Spline Functions and Approximation Theory, Academic Press, New York, 1976, pp. 163-201.

8. F. Richards and I. J. Schoenberg, Notes on spline functions. IV. A cardinal spline analogue of the theorem of the brothers Markov, Israel J. Math. 16 (1973), 94-102.

9. I. J. Schoenberg, Notes on spline functions. III. On the convergence of the interpolating cardinal spline as their degree tends to infinity, Israel J. Math. 16 (1973), 87-93.

10. On the remainders and convergence of cardinal spline interpolation for almost periodic functions, Studies in Spline Functions and Approximation Theory, Academic Press, New York, 1976, pp. 277-303.

Department of Mathematics, The University, Dundee DDl 4HN, Scotland, United Kingdom School of Mathematical Sciences, University Science Malaysia, Penang, Malaysia 